Pacific Journal of Mathematics

COMPLEMENTED CONGRUENCES ON COMPLEMENTED 


\title{
COMPLEMENTED CONGRUENCES ON COMPLEMENTED LATTICES
}

\author{
M. F. JANOWITZ
}

\begin{abstract}
We prove that a congruence relation on a complemented lattice has a complement if and only if it is the minimal congruence generated by a central element. This result is then used to show that a complemented lattice has a Boolean lattice of congruence relations if and only if it is the direct product of a finite number of simple lattices. It is also used to obtain some information on the structure of complemented lattices whose lattice of congruences is a Stone lattice.
\end{abstract}

1. Introduction. What does it mean for a congruence relation $\theta$ on a complemented lattice $L$ to have a complement in the lattice Con $(L)$ of congruence relations of $L$ ? The answer to this question provides the underlying theme for the paper. In case every interval $[0, a]$ is complemented, then some results of Grätzer and Schmidt ([1], Theorem 11, p. 56 and [1], Lemma 8, p. 37) can be used to show that $\theta$ has a complement in Con $(L)$ if and only if there is a central element $z$ of $L$ such that $\theta$ is the minimal congruence generated by the ideal $[0, z]$. In $\S 2$ this result is extended to an arbitrary complemented lattice. It is then used to obtain the structure of those complemented lattices for which $\operatorname{Con}(L)$ is a Boolean algebra. At this point, it is shown (for a suitable class of lattices) that Con $(L)$ being a Stone lattice is related to the existence of certain suprema in $L$.

2. Complemented congruences. Let $\theta, \theta^{\prime}$ be congruences on the bounded lattice $L$. Suppose $\theta, \theta^{\prime}$ are disjoint in that $a\left(\theta \cap \theta^{\prime}\right) b$ implies $a=b$. The key to what is happening is provided by

Lemma 1. Let 0 denote the least element of $L$. If $0<a<b$ with $0 \theta a \theta^{\prime} b$, then:

(1) $(x \vee a) \wedge b=(x \wedge b) \vee a$ for every $x \in L$.

(2) a is neutral in $[0, b]$.

If $L$ is complemented, we may add:

(3) $a$ is central in $[0, b]$.

(4) There is an element $c \in L$ such that $0<c<b$ and $0 \theta^{\prime} c \theta b$.

Proof. (1) Given $x \in L$, we note that $(x \vee a) \wedge b \theta x \wedge b \theta(x \wedge b) \vee a$. Since $(x \vee a) \wedge b,(x \wedge b) \vee a \in[a, b]$ with $a \theta^{\prime} b$, it follows that $(x \vee a) \wedge$ $b=(x \wedge b) \vee a$. 
(2) Let $x, y \in[0, b]$, and set $s=(a \wedge x) \vee(x \wedge y) \vee(y \wedge a), t=$ $(a \vee x) \wedge(x \vee y) \wedge(y \vee a)$. Then $s \theta t$ follows from $0 \theta a$, and $s \theta^{\prime} t$ from $a \theta^{\prime} b$. Consequently, $s=t$, and by [2], $a$ is neutral in [0,b].

(3) Let $a^{\prime}$ be a complement for $a$ in $L$. Then $a \wedge\left(b \wedge a^{\prime}\right)=0$, and by (1), $a \vee\left(b \wedge a^{\prime}\right)=\left(a^{\prime} \vee a\right) \wedge b=b$, so $b \wedge a^{\prime}$ is a complement for $a$ in $[0, b]$. But this says that $a$ is central in $[0, b]$.

(4) Take $c=b \wedge a^{\prime}$.

We are now ready to state our principal result.

Theorem 2. Let $L$ be a complemented lattice. A congruence relation $\theta$ has a complement in $\operatorname{Con}(L)$ if and only if there is a central element $z$ of $L$ such that $\theta$ is the minimal congruence generated by $[0, z]$.

Proof. If $z$ exists, it is clear that $\theta$ has a complement in Con $(L)$. Suppose conversely that $\theta$ has a complement $\theta^{\prime}$ in Con $(L)$. We may then find a finite chain

$$
0=x_{0}<x_{1}<x_{2}<\cdots<x_{n-1}<x_{n}=1
$$

of minimal length such that $x_{i-1} \theta x_{i}$ or $x_{i-1} \theta^{\prime} x_{i}$ for $i=1,2, \cdots, n$. If $n=1$, there is nothing to prove, so we may as well assume $n \geqq 2$. In view of Lemma 1 (4), we may also assume that $x_{0} \theta x_{1} \theta^{\prime} x_{2}$. If $n \geqq 3$ we must have $x_{2} \theta x_{3}$. We may apply Lemma 1 (4) to the interval $\left[0, x_{2}\right]$ to obtain an element $c \in L$ such that $0<c<x_{2}$ and $0 \theta^{\prime} c \theta x_{2}$. But then the chain

$$
0=x_{0}<c<x_{3}<\cdots<x_{n-1}<x_{n}=1
$$

with $0 \theta^{\prime} c \theta x_{3}$ is a chain of shorter length than our original minimal length chain. From this contradiction we deduce that $n=2$, so there is an element $z$ such that $0<z<1$ and $0 \theta z \theta^{\prime} 1$. By Lemma 1, $z$ is central. Evidently $x \theta y$ is equivalent to $x \vee z=y \vee z$, so $\theta$ is the minimal congruence generated by the ideal $[0, z]$.

This leads immediately to

THEOREM 3. Let $L$ be a complemented lattice. A necessary and sufficient condition for $\operatorname{Con}(L)$ to be a Boolean algebra is that $L$ be the direct product of a finite number of simple lattices.

Proof. Sufficiency is clear. To establish necessity, it suffices to show that if $\operatorname{Con}(L)$ is Boolean, then $L$ must have a finite center. For then, if $z_{1}, z_{2}, \cdots, z_{n}$ are the atoms of the center of $L$, and if 
$L_{i}=\left[0, z_{i}\right]$, then $L$ would be isomorphic to the direct product of the irreducible lattices $L_{1}, L_{2}, \cdots, L_{n}$. But each $L_{i}$ is a homomorphic image of $L$, whence each $\operatorname{Con}\left(L_{i}\right)$ is Boolean. An application of Theorem 2 to the complemented lattice $L_{i}$ yields Con $\left(L_{i}\right)$ a 2 element chain, since the center of $L_{i}$ is $\left\{0, z_{i}\right\}$. In other words, each $L_{i}$ is in fact simple.

We now proceed to show the center of $L$ to be finite. Suppose this were not true. We could then find an ideal $J$ of the center of $L$ that is not principal. Define $\theta$ on $L$ by the rule $x \theta y$ iff $x \vee z_{\alpha}=$ $y \vee z_{\alpha}$ for some $z_{\alpha} \in J$, and note that $\theta \in \operatorname{Con}(L)$. But this forces the existence of a central element $z$ such that $x \theta y$ iff $x \vee z=y \vee z$, contrary to the fact that $J$ is not a principal ideal of the center.

3. Stone lattices. In [3] we asked what it meant for Con $(L)$ to be a Stone lattice in the sense that for each congruence relation $\theta, \theta^{*}$ and $\theta^{* *}$ are complements in $\operatorname{Con}(L)$. Here $\theta^{*}$ denotes the pseudocomplement of $\theta$ in $\operatorname{Con}(L)$. The foregoing results can be used to show that for a fairly wide class of complemented lattices, this is related to the existence of certain suprema in $L$. The class of lattices we have in mind is the class that satisfies (A), $\left(\mathrm{A}^{*}\right),(\mathrm{B})$ and $\left(\mathrm{B}^{*}\right)$ of [4]. (Note: Axiom $\left(X^{*}\right)$ denotes the dual of Axiom $X$.) For the reader's convenience we restate $(\mathrm{A})$ and $(\mathrm{B})$ here:

(A) $a / 0 \longrightarrow c / d$ with $c>d$ implies $c / d \longrightarrow a_{1} / a_{2}$ for suitable $a_{1}, a_{2}$ such that $a \geqq a_{1}>a_{2}$

(B) $a>b$ implies the existence of an element $t$ such that $t \theta_{a / b} 1, t \geqq a$.

It should be noted that $\theta_{a / b}$ denotes the smallest congruence that identifies $a$ and $b$. To illustrate the scope of these axioms, we mention that $(\mathrm{A}),\left(\mathrm{A}^{*}\right),(\mathrm{B})$ and $\left(\mathrm{B}^{*}\right)$ are satisfied by each of the following types of lattices:

(i) any bounded relatively complemented lattice;

(ii) any lattice that is both atomistic and dual atomistic;

(iii) any uniquely complemented lattice;

(iv) any simple lattice;

( $v$ ) the direct product of lattices of any of the preceding types.

Here then is our result.

THEOREM 4. (1) Let $L$ be a complemented lattice that satisfies $\left(\mathrm{A}^{*}\right)$ and $\left(\mathrm{B}^{*}\right)$. If $\operatorname{Con}(L)$ is a Stone lattice, then the kernel of every congruence relation of $L$ has a supremum in $L$.

(2) Let $L$ be a bounded lattice satisfying (A), (A*), (B) and $\left(\mathrm{B}^{*}\right)$. If the kernel of each congruence relation of $L$ has a supremum in $L$, then $\operatorname{Con}(L)$ is a Stone lattice. 
Proof. (1) Let $\theta \in \operatorname{Con}(L)$ have kernel $J$. By the dual of Theorem 2, there is a central element $z$ of $L$ such that $\theta^{*}$ is the minimal congruence generated by the filter $[z, 1]$. By the dual of [4], Theorem 3, p. 179, $a \theta^{*} 1$ iff $a$ is an upper bound for the kernel of $\theta$. Hence $z=\vee J$.

(2) Let $\theta \in \operatorname{Con}(L)$ and let $z$ be the supremum of the kernel of $\theta$. By the dual of [4], Theorem 3, p. 179, [z, 1] $=\left\{t \in L: t \theta^{*} 1\right\}$. Since $z$ is a lower bound for $\left\{t \in L: t \theta^{*} 1\right\}$, we may apply [4], Theorem 3 , p. 179 with $\theta$ replaced by $\theta^{*}$ to deduce that $z \theta^{* *} 0$. Thus, $0 \theta^{* *} z \theta^{*} 1$ and so $\theta^{* *}$ is a complement for $\theta^{*}$ in $\operatorname{Con}(L)$.

Corollary. For $L$ a Boolean algebra, Con $(L)$ is a Stone lattice if and only if $L$ is complete.

Proof. Suppose Con $(L)$ is a Stone lattice. Then for $S$ an arbitrary nonempty subset of $L$, the ideal $J$ generated by $S$ is the kernel of a congruence. Hence $\vee J$ exists in $L$, and it is clearly effective as the supremum of $S$. The converse is clear.

In conclusion, the author would like to express his gratitude to the referee for providing more efficient proofs of some of the results, and in particular, for suggesting the present version of Theorem 4.

\section{REFERENCES}

1. G. Grätzer and E. T. Schmidt, Standard ideals in lattices, Acta Math. Acad. Sci. Hung., 16 (1965), 289-301.

2. G. Grätzer, A characterization of neutral elements in lattices, Magyar Tud. Akad. Mat. Kutuó int. Közl., 7 (1962), 191-192.

3. M. F. Janowitz, Projective ideals and congruence relations, Univ. of New Mexico Tech. Report No. 51 (1964).

4._- On a paper by Iqbalunissa, Fund. Math., 78 (1973), 177-182.

Received June 17, 1976 and in revised form May 3, 1977. Presented in part to the Society April 15, 1977.

UNIVERSITY OF MASSACHUSETTS

AMHerst, MA 01002 


\section{PACIFIC JOURNAL OF MATHEMATICS}

EDITORS

RICHARD ARENS (Managing Editor)

University of California

Los Angeles, California 90024

C. W. Curtis

University of Oregon

Eugene, OR 97403

C. C. MOORE

J. DUGUNDJI

Department of Mathematics University of Southern Californa Los Angeles, California 90007

R. Finn AND J. Milgram Stanford University Stanford, California 94305

University of California

Berkeley, CA 94720

\section{ASSOCIATE EDITORS}

E. F. BeCKENBACH

B. H. NeUMANN

F. WOLF

K. YosHida

\section{SUPPORTING INSTITUTIONS}

UNIVERSITY OF BRITISH COLUMBIA UNIVERSITY OF SOUTHERN CALIFORNIA CALIFORNIA INSTITUTE OF TECHNOLOGY UNIVERSITY OF CALIFORNIA MONTANA STATE UNIVERSITY UNIVERSITY OF NEVADA, RENO STANFORD UNIVERSITY UNIVERSITY OF TOKYO UNIVERSITY OF UTAH NEW MEXICO STATE UNIVERSITY WASHINGTON STATE UNIVERSITY OREGON STATE UNIVERSITY UNIVERSITY OF WASHINGTON UNIVERSITY OF OREGON OSAKA UNIVERSITY 


\section{Pacific Journal of Mathematics \\ Vol. 73, No. 1 \\ March, 1977}

Thomas Robert Berger, Hall-Higman type theorems. $V \ldots \ldots \ldots \ldots \ldots \ldots \ldots$

Frank Peter Anthony Cass and Billy E. Rhoades, Mercerian theorems via

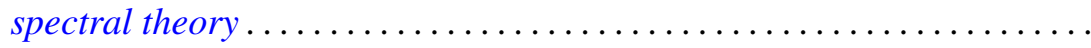

Morris Leroy Eaton and Michael David Perlman, Generating $\mathrm{O}(n)$ with

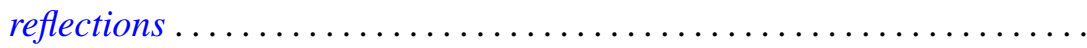

Frank John Forelli, Jr., A necessary condition on the extreme points of a

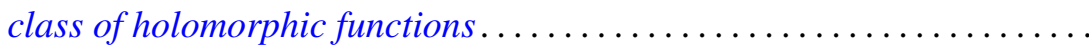

Melvin F. Janowitz, Complemented congruences on complemented

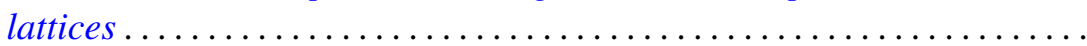

Maria M. Klawe, Semidirect product of semigroups in relation to amenability, cancellation properties, and strong $F \phi$ lner conditions....

Theodore Willis Laetsch, Normal cones, barrier cones, and the "spherical image" of convex surfaces in locally convex spaces ................

Chao-Chu Liang, Involutions fixing codimension two knots.............

Joyce Longman, On generalizations of alternative algebras .............

Giancarlo Mauceri, Square integrable representations and the Fourier

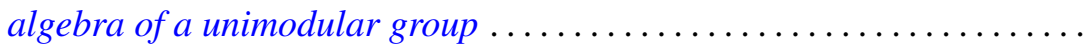

J. Marshall Osborn, Lie algebras with descending chain condition...

John Robert Quine, Jr., Tangent winding numbers and branched

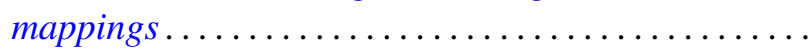

Louis Jackson Ratliff, Jr. and David Eugene Rush, Notes on ideal covers and associated primes .

H. B. Reiter and N. Stavrakas, On the compactness of the hyperspace of faces.

Walter Roth, A general Rudin-Carlson theorem in Banach-spaces ..

Mark Andrew Smith, Products of Banach spaces that are uniformly rotund in every direction.

Roger R. Smith, The R-Borel structure on a Choquet simplex ...

Gerald Stoller, The convergence-preserving rearrangements of real infinite series. ...

Graham H. Toomer, Generalized homotopy excision theorems modulo a Serre class of nilpotent groups...

Norris Freeman Weaver, Dehn's construction and the Poincaré conjecture....

Steven Howard Weintraub, Topological realization of equivariant intersection forms... 